
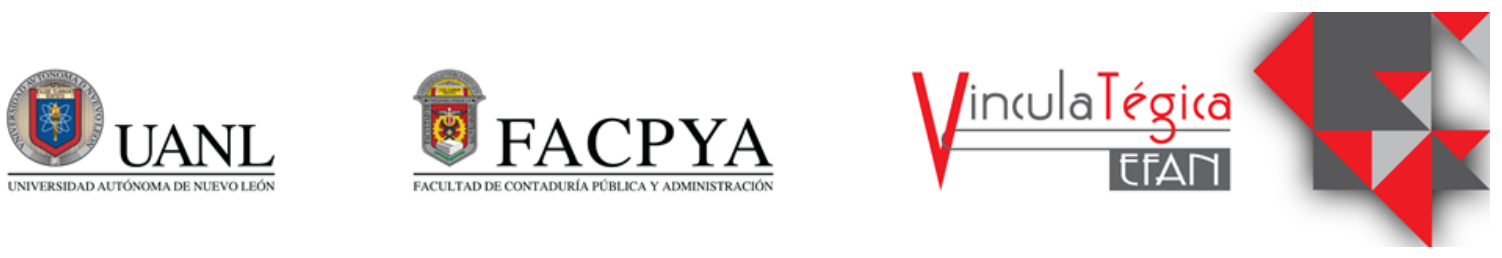

\title{
Buenas prácticas en tiempos de pandemia, caso material digital para la tutoría entre pares
}

\author{
Norma Martínez Álvarez ${ }^{1}$ y Luis Gerardo Rodríguez Bermea ${ }^{2}$ \\ ${ }^{1}$ Universidad Autónoma de Nuevo León, norma.martinezal@uanl.edu.mx, Ave. Universidad S/N Col. Ciudad \\ Universitaria, $(+52) 8183204080$ \\ ${ }^{2}$ Universidad Autónoma de Nuevo León, lrodriguezb@uanl.edu.mx, Ave. Universidad S/N Col. Ciudad \\ Universitaria, $(+52) 8183204080$
}

\begin{abstract}
Información del artículo revisado por pares
Fecha de aceptación: junio-2021

Fecha de publicación en línea: diciembre-2021

DOI: https://doi.org/10.29105/vtga7.1-164
\end{abstract}

\begin{abstract}
Resumen
El objetivo del presente trabajo es describir un Estudio de caso sobre el proceso de producción de material didáctico digital, como estrategia emergente para migrar a la modalidad virtual de un Programa de Tutoría entre pares de la Facultad de Contaduría Púbica y Administración de la Universidad Autónoma de Nuevo León, en el ciclo Junio - Diciembre del 2020. En un contexto de aislamiento social, suspensión de clases presenciales, acelerado cambio a la educación remota y ruptura de todo esquema acostumbrado debido a la pandemia, esta estrategia iniciada por dos personas contó con la oportunidad que surge ante la crisis, de construir una gran empresa al sumar la participación de 445 estudiantes universitarios. La investigación realizada fue cualitativa, el método estudio de caso, de tipo descriptivo y transversal. Las proposiciones teóricas que se utilizaron fueron: Tutoría entre pares, Trabajo colaborativo y Principios de buenas prácticas. Los instrumentos que se aplicaron: el Focus group, los registros de archivo, la entrevista directa y la observación participante. Los resultados la producción de 74 Manuales digitales de ejercicios prácticos y videos tutoriales de aprendizaje para la carrera de Contador Público y 105 Manuales digitales de ejercicios prácticos $\mathrm{y}$ videos tutoriales de aprendizaje para la carrera de Licenciado en
\end{abstract}

Administración. El aporte, ampliar la información de estrategias académicas y buenas prácticas en tiempos del COVID-19.

Palabras clave. Buenas prácticas, COVID-19, Estrategias académicas, Tutoría en pares.

Códigos JEL: I20, I21, I29

\begin{abstract}
The aim of this work is to describe a case study on the process of producing digital teaching material, as an emergent strategy to migrate from a Peer Tutoring Program to virtual mode of the Facultad de Contaduría Pública y Administración at Universidad Autonoma de Nuevo Leon for the period from June through December 2020. This two-person strategy had the opportunity in the face of an emerging crisis to build a large company by adding the participation of 445 university students, in a context of social isolation, suspension of faceto-face classes, an accelerating rate of change to remote education and the idea of breaking established patterns by the pandemic. The study presents the results of a qualitative research and the case study method was descriptive and transversal. The theoretical propositions used were: Peer Tutoring, Collaborative Work, and Principles of Good Practice. The instruments that were applied: focus group, file records, direct interview and participating observation. The
\end{abstract}


results: the production of 74 Digital Manuals of Practical Exercises and Tutorials Learning Videos for the career in Public Accounting and 105 Digital Manuals of Practical Exercises and Tutorial Videos for the Bachelor in Administration. The contribution, expand the information of academic strategies and good practices in COVID-19 times.

Keywords. Academic strategies, COVID-19, Good practices, Peer tutoring.

JEL Codes: I20, I21, I29

\section{INTRODUCCIÓN}

La Organización Mundial de la Salud (OMS) en febrero del 2020 nombra oficialmente la enfermedad por coronavirus como COVID19 , la cual se convierte en un gran problema de salud pública y una amenaza global por su rápida propagación. (Apaza, Seminario y Santa Cruz, 2020). Esta pandemia sacudió a la población mundial y generó una crisis sin precedentes en salud y en todos los ámbitos. En el área de la educación, esta contingencia ha dado lugar al cierre masivo de actividades presenciales de las instituciones educativas a fin de evitar la propagación del virus y mitigar sus impactos. (Ruiz, 2020).

Los sistemas educativos en general han recurrido a la rápida transición a la educación remota, a distancia o virtual mediante la utilización de una diversidad de formatos y plataformas virtuales que han requerido grandes esfuerzos tanto institucionales como de los profesores y de los estudiantes para lograr la continuidad, calidad y efectividad de los procesos de aprendizaje, cambios que parecen ser funcionales, aunque sus resultados aún no son totalmente perceptibles y requieren registros posteriores de evaluación (Luján, 2020).

En este contexto de aislamiento social, suspensión de clases presenciales, acelerado cambio a la educación remota y ruptura de todo esquema acostumbrado debido a la pandemia, las acciones que iniciaron este estudio partieron de la necesidad del Programa de Tutoría entre pares de nuestra Facultad de emigrar a la modalidad virtual, para continuar la labor de apoyo al desarrollo académico de los estudiantes universitarios. La tutoría entre pares consiste en que compañeros voluntarios de diferente edad y avance académico, tras un proceso de formación, en un marco de relación asimétrico estudiante tutor $\mathrm{y}$ estudiante tutorado, planificado y supervisado por profesionales, facilitan ayuda y apoyo para mejorar el rendimiento académico. (Topping, 2015).

El estudiante tutor al igual que todo estudiante, se ha visto abrumado por los cambios académicos ocasionados por la pandemia, impactando al Programa con una baja del $20 \%$ de sus estudiantes tutores voluntarios. Ante esta situación se formula la estrategia: producción de material digital, para ponerlo a disposición del estudiante tutor y con esto aligerar la tarea de digitalización de los contenidos para las asesorías. Lo anterior tomando en cuenta lo que señalan Duran y Estay-Niculcar (2016) que en la educación virtual el material digital y multimedia cobran una especial relevancia ya que el proceso de aprendizaje de los estudiantes está guiado, en su mayor parte por ellos. Aquí surge la pregunta de investigación: $\mathrm{Si}$ el personal disponible solo contempla dos administrativos del Programa y dos prestatarios de servicio social, ¿Cómo producir el material digital necesario para la tutoría entre pares, modalidad virtual?

El presente trabajo tiene por objetivo describir un Estudio de caso sobre el proceso de producción de material digital, como estrategia emergente para migrar a la modalidad virtual de un Programa de Tutoría entre pares de la Facultad de Contaduría Púbica y Administración (FACPYA) de la Universidad Autónoma de Nuevo León, (UANL) en el ciclo Junio - Diciembre del 2020. Las proposiciones teóricas que se utilizan: Tutoría entre pares, Trabajo colaborativo y Principios de buenas prácticas. El aporte, ampliar la información de estrategias académicas y buenas prácticas en tiempos del COVID-19.

\section{MARCO TEÓRICO}

\subsection{Tutoría entre pares}

Los Programas de tutoría entre pares en las instituciones de educación superior se establecen y funcionan regularmente como un servicio de apoyo al estudiante en la búsqueda de desarrollo, mediante la didáctica tutoría 
entre pares, que Topping (2015) define como la ayuda proporcionada por compañeros más avanzados, que tras un proceso de formación y la guía del profesor, en una relación de tutor, tutorado proporcionan asesoría y orientación a los estudiantes para mejorar su rendimiento académico.

La planeación e implementación de un Programa de Tutoría entre pares requiere el diseño, la planificación y la evaluación de un plan. Viafra y Ariza (2008) describen que las etapas implican: detección de las necesidades, definir objetivos, seleccionar contenidos, prever recursos, vigilancia o monitoreo y constante revisión de la experiencia.

De acuerdo a Mkpanang (2016) la metodología utilizada en la tutoría entre pares, emerge de la tutoría instruccional y sucede, cuando profesionales dirigen a los estudiantes como medio para el aprendizaje de otros estudiantes. Utiliza métodos de aprendizaje colaborativo y se basa en varias corrientes educativas: Teoría genética de Piaget, Teoría sociocultural de Vygotsky y el modelo pedagógico constructivista. (Menéndez, 2011).

Las investigaciones sobre la Tutoría entre pares la señalan como una metodología efectiva complementaria al proceso de aprendizaje, que da evidencia de resultados positivos en distintas áreas como: rendimiento académico, mejora de habilidades psicosociales, responsabilidad sobre el propio aprendizaje y fortalecimiento de la autoestima tanto para el estudiante tutorado como para el estudiante tutor. (Topping, 2015; Arco y Fernández, 2011).

En la estrategia pedagógica Tutoría entre pares, los estudiantes son los protagonistas en el proceso de aprendizaje, tanto los tutorados como el tutor, pues tienen un rol activo en todos los aspectos académicos como en los psicosociales. (Topping, 2015). Otro factor importante en esta metodología es el Profesor como guía del estudiante tutor, quien le proporciona en diferentes momentos del proceso de enseñanza-aprendizaje, las bases y herramientas requeridas para su labor pedagógica. (Cardozo, 2011).

El tutor par es un estudiante con un alto rendimiento académico y de semestres superiores, conocedor de un área específica, quien de manera voluntaria se inscribe para participar en un Programa de Tutoría entre Pares. (Torrado et. al, 2016). El Tutor par, además de apoyar a sus tutorados también se beneficia al participar en la tutoría entre pares pues fortalece su conocimiento previo y al estar comprometido en su labor hace un aporte pedagógico y social muy importante para su institución. (Duran y Flores, 2015).

\subsection{Educación virtual}

La educación virtual ha sido definida como la educación a distancia a través del ciberespacio, posible mediante la conexión y uso de internet, que no necesita de un tiempo y espacio específicos, que permite establecer un nuevo escenario de comunicación entre docentes y estudiantes (Bonilla, 2016). De acuerdo a Silvio (2006) la educación virtual se basa en un modelo cooperativo donde interactúan los participantes haciendo uso de la tecnología de la Información y comunicación, principalmente la internet y servicios asociados.

Las tecnologías de la Información y comunicación han promovido la virtualización de la educación superior como un modelo enseñanza - aprendizaje que incorpora la tecnología y la adquisición de competencias demandadas en el campo laboral. (Tunnermann, 2008; Rama, 2014).

La modalidad virtual se distingue por mayor autonomía e independencia de los estudiantes en el desarrollo de su proceso de aprendizaje al marcar su propio ritmo de trabajo, lo que le exige mayor autorregulación, responsabilidad y compromiso. (Suárez y Anaya, 2004). Es un proceso interactivo que promueve el análisis y discusión de los contenidos a través de medios sincrónicos (aula virtual, videoconferencias) y medios asincrónicos (foros, correo electrónico) en una relación dialógica. (Pérez, et al., 2006).

Duran y Estay-Niculcar (2016) señalan que en la educación virtual el material o recursos didácticos multimedia cobran una especial relevancia ya que el proceso de aprendizaje de los estudiantes está guiado, en su mayor parte por ellos. Estos se distinguen 
por presentar contenido informativo a los estudiantes mediante diversos formatos: documentos textuales, hipertextos, presentaciones multimedia; ofrecer actividades variadas que desarrollen experiencias de aprendizaje de los contenidos; y contar con procesos comunicativos variados, fluidos y constantes con el profesor y sus pares a lo largo de la asignatura. (De Pablo y Jiménez, 2007).

Las primeras experiencias de la educación virtual se registraron en la década de los setenta en Inglaterra con la Open University, y posteriormente en España en la Universidad Nacional de Educación a Distancia (UNED) y en Alemania en la FernUniversitat. (García, 1999). Para la década de los ochentas incursionaron en la educación virtual países como Irlanda, Francia, Portugal y Noruega. En América Latina para los noventas países como México, Panamá y Brasil. (Fernández y Vallejo, 2014).

La educación virtual ha sido tema de interés en la educación superior como estrategia en iniciativas por ejemplo el Proyecto sobre Educación Superior Virtual y a Distancia por parte del Instituto Internacional de la UNESCO para la educación superior en América Latina y el Caribe en el año 2002 (ANUIES - UNESCO, 2004). Además, ha hecho posible el cambio de paradigma de gestión del conocimiento centrado en la enseñanza, hacia la educación centrada en el estudiante. (Coll, 2008).

Con la modalidad virtual se han logrado modelos más flexibles de enseñanzaaprendizaje, creación de escenarios tecnológicos educativos que transforman el modelo educativo de la universidad tradicional a un nuevo modelo de universidad virtual. (Gros, 2012).

Para Marciniak y Gairín-Sallán (2018) una modalidad de educación virtual de calidad, debe contemplar ciertos requisitos como: contar con los recursos tecnológicos adecuados y el servicio necesario para acceder al programa educativo; que la estructura y el contenido del curso virtual ofrezcan un valor formativo; que se realicen aprendizajes efectivos y que sea un ambiente satisfactorio tanto para los estudiantes como para los profesores.

Además, para asegurar su calidad una educación virtual debe evaluar de manera permanente la planificación de sus procesos: su base tecnológica para el desarrollo de planes y programas virtuales, el número de estudiantes atendidos, la disponibilidad de material instruccional, las competencias de los profesores, calidad y pertinencia de las estrategias didácticas y la interacción estudiante - profesor y entre pares. (Rodríguez Fernandez, 2014).

\subsection{Buenas prácticas}

Las buenas prácticas son experiencias reales que han sido documentadas $y$ concentran una serie de datos prácticos. Tienen su origen en el sector empresarial y se utilizan para calificar una actividad que ofrece buenos resultados en el contexto en que se realiza y supone el logro de resultados eficaces y eficientes. (De Pablo y González, 2007). La identificación de buenas prácticas, permite aprender de las mismas, contextualizarlas y mejorar las propias.

Epper y Bates (2004) señalan que las buenas prácticas deben mejorar los resultados de un proceso, ser una experiencia sistematizada, documentada, aplicar métodos innovadores y ser extrapolables a otros contextos. Luzón et al. (2009) describe las buenas prácticas como calificativo de cualquier actividad que ofrece buenos resultados en su contexto. Murray (1997) considera que las buenas prácticas representan una estrategia que contribuye a maximizar la efectividad de la empresa y la ubica en la posibilidad de transferencia del conocimiento.

Las buenas prácticas no resultan ajenas a ningún sector, en el ámbito educativo según Sallis (2014) la buena práctica universitaria es una experiencia, programa o proyecto que favorece significativamente a la institución en su aspecto social, cultural, medioambiental o económico. En el campo docente las buenas prácticas son un conjunto de acciones que facilitan el desarrollo de actividades de aprendizaje que logren objetivos formativos previstos y de alto valor.

Con respecto al alcance o potencia de las buenas prácticas docentes Rodríguez 
(2012) lista una serie de indicadores: Permiten la generación de un aprendizaje significativo, involucran a los participantes en las actividades, requieren mayor cantidad de operaciones mentales, trabajo colaborativo y aprendizaje autónomo, promueven el pensamiento divergente, intervienen diferentes campos de conocimiento, establecen interconexiones entre los actores, favorecen el uso de las tecnologías y exigen una evaluación continua.

El tema de buenas prácticas docentes ha inspirado a diferentes autores a desarrollar Modelos originalmente para la educación presencial que incorporan rasgos que hacen identificables las buenas prácticas, destacándose las iniciativas de Chickering y Gamson (1987), Alexander (1997) y Coffield y Edward (2009).

El Modelo de Chickering y Gamson (1987) postula siete principios de buenas prácticas educativas a través de los cuales se ha asistido a la comunidad académica y a las instituciones de educación superior. (Graham et al., 2001) El Modelo de Alexander (1997) establece que una buena práctica es creada en contextos singulares a partir de las ideas y las acciones de profesores y alumnos, utiliza propuestas pedagógicas de manera reflexiva. El Modelo de Coffield y Edward (2009) acentúa la importancia del contexto, el curriculum, la pedagogía, la evaluación, la gestión y la sociedad. (Muñoz 2009).

Los principios que fundamentan las buenas prácticas de acuerdo al Modelo de Chickering y Gamson (1987) son:

- Principio 1: Promover las relaciones entre docentes y alumnos.

- Principio 2: Desarrollar dinámicas de cooperación entre los alumnos.

- Principio 3: Aplicar técnicas activas para el aprendizaje.

- Principio 4: Permitir procesos de retroalimentación.
- Principio 5: Enfatizar el tiempo de dedicación a la tarea.

- Principio 6: Comunicar altas expectativas.

- Principio 7: Respetar la diversidad de formas de aprendizaje.

De acuerdo a Duran y Estay-Niculcar (2016) el Modelo de Chickering y Gamson (1987) ha sido aplicado a numerosos estudios en el campo organizacional y educativo incluyendo estudios sobre la educación virtual entre ellos: Grahan, Cagiltay, Lin, Craner y Duffy (2001); Hutchins (2003); Bangert (2004), Tobin (2004), Dixon (2012); Babb, Stewart y Johnson (2013) y Cakiroglu (2014).

\section{METODOLOGÍA}

La investigación realizada fue cualitativa, el diseño estudio de caso, de tipo descriptivo y transversal. Según Stake (2005) el estudio de caso es el estudio de la particularidad y complejidad de un caso singular para llegar a comprender su actividad en circunstancias particulares. Su objetivo básico es comprender el significado de una experiencia. (Pérez Serrano, 1994).

De acuerdo a Levin y Rubin (1996) la población se define como un conjunto de todos los elementos que estamos estudiando, acerca de los cuales intentamos sacar conclusiones. La población la formaron estudiantes prestatarios de servicio social de la carrera de Contador Público, y Licenciado en Administración, en FACPYA, UANL en el ciclo académico Junio - Diciembre de 2020. El servicio social se entiende como el conjunto de actividades teóricas y prácticas de carácter temporal, que ejecutan y prestan los pasantes y estudiantes en beneficio de la sociedad, el Estado y la comunidad universitaria (Reglamento de Servicio Social UANL, 2014).

Tabla 1. Distribución de la muestra

\begin{tabular}{lcc}
\hline \multicolumn{1}{c}{ Carrera } & Prestatarios de servicio social & \% \\
\hline Contador Público & 188 & 42 \\
Licenciado en Administración & 257 & 58 \\
Total & 445 & 100 \\
\hline
\end{tabular}

Fuente: elaboración propia 
La muestra tuvo carácter no probabilístico, el cual de acuerdo a Pineda et. al., (1994) toma los casos o unidades que estén disponibles en un momento dado y quedó conformada por 445 estudiantes que cubrieron el requisito académico de un promedio igual o superior a 85 y que fueron asignados por la Coordinación de Servicio Social de la dependencia como prestatarios de servicio social al Programa de Tutorías entre Pares. Tabla 1.

Los principales instrumentos y técnicas que se utilizaron para la obtención de la información fueron: El Modelo de Chickering y Gamson (1987) y fuentes varias como: el focus group que de acuerdo a Taylor y Bogdan (2002) es un procedimiento del ámbito social que utiliza el ejercicio de pregunta y respuesta para generar un diálogo y reflexionar un fenómeno desde la perspectiva del actor, es decir en base a su experiencia para definir acciones. También se aplicó la revisión documental, el registro de archivo, entrevistas directas y observación participante. El análisis estadístico se realizó a través de tablas de cálculo utilizando el programa Microsoft Excel para Windows.

\subsection{Estudio de Caso: Material digital para la tutoría entre pares.}

Las acciones que iniciaron este estudio partieron de las disposiciones oficiales debido a la pandemia, sobre la suspensión de clases presenciales y la necesidad del Programa de Tutoría en pares de emigrar a la modalidad virtual, para continuar la labor de apoyo al desarrollo académico de los estudiantes de nuestra Facultad a través de su grupo de tutores pares, estudiantes voluntarios de alta dedicación y desempeño (Topping, 2015) que ante la incertidumbre de la nueva situación académica y la extensión de tiempo requerido para la adaptación de sus materiales de asesoría, más sus altos estándares de desempeño, se vieron abrumados lo cual dio como resultado que un $20 \%$ del grupo decidiera no continuar con su voluntariado.

Ante esta situación se formuló la estrategia emergente de producir material digital para la asesoría y con esto aligerar la tarea del estudiante tutor par en la digitalización de contenidos para las prácticas, ya que como señalan Duran y Estay-Niculcar (2016) en la educación virtual el material didáctico multimedia cobra una especial relevancia pues el proceso de aprendizaje de los estudiantes está guiado en su mayor parte por ellos. Aquí surgió la pregunta de investigación: Si el personal disponible solo contempla dos administrativos del Programa y dos prestatarios de servicio social, con experiencia como tutor par, previstos para el ciclo Junio - Diciembre de 2020. ¿Cómo producir el material digital necesario para la tutoría entre pares, modalidad virtual?

Nuevas disposiciones institucionales surgen, a consecuencia de la pandemia de COVID-19 dictando que los prestatarios de servicio social realizarán sus actividades en modalidad virtual y en su dependencia, (FACPYA, UANL 2020) lo que presenta la oportunidad en la crisis y este Proyecto del Programa de Tutoría en Pares se postula a la coordinación correspondiente, como una actividad investigativa, académica y administrativa de acuerdo al Reglamento de Servicio Social, UANL (2014) y es aceptado para un mayor número de asignaciones en el ciclo antes mencionado.

Por el enfoque académico de la participación se extendió el requisito de tener una buena trayectoria académica y un promedio igual o superior a 85. Así la resolución fue la asignación de 188 estudiantes de la carrera de Contador Público y 257 de la carrera de Licenciado en Administración, sumando un total de 445 prestatarios de servicio social asignados al Programa de tutoría entre pares.

Con este gran potencial al alcance, mediante focus group con la participación de dos administrativos y los dos prestatarios previstos para el ciclo Junio - Diciembre 2020, se inició la planeación del proyecto tomando 
como base las proposiciones teóricas siguientes: Tutoría entre pares, Trabajo colaborativo y Principios de buenas prácticas de acuerdo al Modelo de Chickering y Gamson (1987) que son:

Promover

relaciones

entre Tutores

pares líderes

con el resto de

los estudiantes;

desarrollar

dinámicas de

colaboración;

aplicar técnicas

activas; contar

con procesos Fuente: elaboración propia

de

retroalimentación; enfatizar tiempo dedicado a

la tarea; comunicar altas expectativas; y respetar la diversidad. Los objetivos: Producción de manuales de ejercicios prácticos digitales y videos tutoriales de aprendizaje para el Programa de Tutoría en pares, de FACPYA, UANL.

Por la naturaleza de los contenidos de las materias prácticas, se decidió separar los equipos de trabajo por carrera, y tener una cobertura de primero a quinto semestre, en base a los programas analíticos vigentes de cada materia, para lo cual se solicitó el cronograma respectivo a la dependencia

En la etapa de organización se definió la estructura para cada carrera de la manera siguiente: Consejo administrativo, Tutores pares líderes de carrera, Tutores pares líderes de semestre, Tutores pares líderes de turno, y Prestatarios de servicio social de producción de manuales de ejercicios prácticos y videos tutoriales de aprendizaje, en equipos de 2 a 3 personas en turnos matutino, vespertino y nocturno, a quienes se les establecieron dos sesiones virtuales de trabajo colaborativo a la semana con duración de 2 horas y un acceso a Drive para cargar los avances de producción mediante correo electrónico.

Funciones a desarrollar por cargo:

- Consejo administrativo: planeación, organización, dirección y control, operativa y estratégica, del proyecto Material digital para tutoría entre pares en el ciclo Junio Diciembre de 2020.

- Tutores pares líderes de carrera: reportes continuos al Consejo

administrativo; monitoreo de Tutores pares líderes de semestre y de turno y tercer filtro de calidad en la revisión de avances de producción.

- Tutores pares líderes de semestre: reporte continuo al Tutor par líder de carrera; reuniones de seguimiento con Tutores pares líderes de turno; segundo filtro de calidad en la revisión de avances de producción; reportes de asistencia mensual y carga en Drive de avances para revisión.

correspondiente y se definió el programa de actividades de acuerdo a la tabla 2.

- Tutores pares líderes de turno: reporte continuo al Tutor par líder de semestre; reuniones de seguimiento con equipos de producción por turno; primer filtro de calidad en la revisión de avances de producción; reportes de asistencia diaria y carga en Drive de avances para revisión.

- Equipos de producción de manuales de ejercicios prácticos y videos tutoriales de aprendizaje por turno matutino, vespertino y nocturno: asistencia a sesiones virtuales de trabajo colaborativo en el horario asignado; elaboración de manuales de ejercicios mediante investigación, estudio del tema asignado de acuerdo al formato y a la rúbrica señalada y elaboración de un video tutorial de aprendizaje sobre el tema asignado basado en 
investigación y estudio del tema de acuerdo al formato y a la rúbrica señalada.

En la etapa de dirección, el Consejo administrativo a través de focus group, estableció los canales de comunicación para dar a conocer a todos los prestatarios de servicio social participantes los objetivos del proyecto, el programa de actividades y los formatos y las rúbricas para la elaboración de cumplimiento de 480 horas de acuerdo al Reglamento de Servicio Social, UANL (2014).

- Formato de Manuales de Ejercicios Prácticos.

Introducción: un breve desglose de por qué es importante el Manual de ejercicios prácticos en el fortalecimiento del conocimiento.

Mostrar por lo menos 3 objetivos específicos

Tabla 3. Sesiones virtuales de trabajo colaborativo

\begin{tabular}{lclc}
\hline \multicolumn{1}{c}{ Semestre } & \multicolumn{1}{c}{ Días } & \multicolumn{1}{c}{ Turno } & Horario \\
\hline Primero & Lunes y Miércoles & Matutino, vespertino y nocturno & 10:00, 15:00 y 19:00 \\
Segundo & Martes y Jueves & Matutino, vespertino y nocturno & 10:00, 15:00 y 19:00 \\
Tercero & Lunes y Miércoles & Matutino, vespertino y nocturno & 10:00, 15:00 y 19:00 \\
Cuarto & Martes y Jueves & Matutino, vespertino y nocturno & 10:00, 15:00 y 19:00 \\
Quinto & Lunes y Miércoles & Matutino, vespertino y nocturno & 10:00, 15:00 y 19:00 \\
\hline
\end{tabular}

Fuente: elaboración propia

los manuales y los videos tutoriales de aprendizaje, a través de investigación, revisión documental y estudio del tema, lo cual se dio a conocer en la reunión inicial del 16 de Junio en tres turnos, matutino, vespertino $\mathrm{y}$ nocturno.

Las actividades para ejercer liderazgo y mantener la motivación mediante entrevistas directas y el monitoreo permanente a través de la observación participante se iniciaron el día 22 de Junio con sesiones virtuales semanales de tutoría entre pares y trabajo colaborativo entre Tutores pares líderes de turno y equipos de producción. Sesiones virtuales de trabajo

En la etapa de control mediante observación participante, entrevista directa y registros de archivo se dio seguimiento a los estándares, formatos y rúbricas estipuladas desde la planeación del Proyecto Material digital para la tutoría entre pares modalidad virtual, los cuales se comentan a continuación:

- Registros de asistencia a cargo de Tutores pares líderes de turno con los equipos de producción en las sesiones semanales virtuales, Tutores pares líderes de semestre, Tutores pares líderes de carrera a Consejo administrativo de forma mensual para turnarlos a la coordinación de Servicio Social de la dependencia para el registro de del tema.

Escribir todos los conceptos básicos del tema asignado, de acuerdo con diferentes autores, mínimo 3 menciones bibliográficas por concepto.

colaborativo por carrera entre Tutor par líder de carrera y Tutores pares líderes de semestre efectuándose el segundo y último viernes de cada mes. Tutor par líder de carrera y Tutores pares líderes de turno el segundo martes de cada mes, durante los meses de Julio, Agosto, Septiembre, Octubre y Noviembre de 2020, como se muestra en la Tabla 3. prácticos.

- Rúbrica de Manuales de ejercicios

1. Portada Oficial.

2. Nombre de los autores del Manual de ejercicios prácticos.

3. Fecha de creación.

4. Índice por temas.

5. Nota de agradecimiento a los colaboradores de elaboración.

6. Introducción. Objetivos del Manual de ejercicios prácticos.

7. Contenido: Teoría y ejemplo desarrollado. Ejercicios: planteamiento del problema, cuadro 
de procedimiento y cuadro de solución.

8. Bibliografía.

Apéndice: Autoevaluación.

- Tipografía

Letra Arial 12 para el contenido.

Márgenes Normales.

Interlineado de 1.15 .

Títulos en letra Arial 22 y negritas, color negro.

En caso de subtítulos, letra Arial 18, color negro, usando el formato establecido por el programa

Word.

Bibliografías en formato APA. Figura 1

Figura 1. Portada Manual de ejercicios prácticos

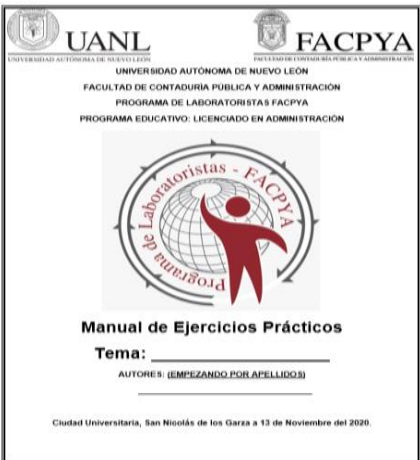

Fuente: elaboración propia
- Formato de Video tutorial de aprendizaje.

Introducción al video tutorial: una breve explicación del por qué es importante.

Mencionar el objetivo específico del tema.

Mencionar los conceptos básicos del tema de forma breve.

Explicar por lo menos 3 problemas del Manual de Ejercicios Prácticos, respondiendo a las preguntas "¿Qué hacer?, ¿Cómo hacerlo?, ¿Cuándo hacerlo? y ¿Por qué hacerlo?”, salvo previa consulta por necesidad del tema. Para las materias de Contabilidad solo un problema. El video tutorial debe tener una duración de 20 minutos, salvo previa consulta por necesidad del tema.

Mencionar las fuentes bibliográficas.

- Rúbrica de Video tutorial de aprendizaje.

1. Portada oficial.

2. Presentación (Presentación del expositor del video).

3. Introducción. Objetivo del tema.

4. Conceptos básicos.

5. Problemas respondiendo a las preguntas "¿Qué hacer?, ¿Cómo hacerlo?, ¿Cuándo hacerlo? y ¿Por qué hacerlo?"

6. Conclusión general del tema.

7. Fuentes bibliográficas.

- Forma de entrega de manuales de ejercicios prácticos y videos tutoriales de aprendizaje.

Tabla 4. Programación de revisión de avances

\begin{tabular}{lcccc}
\hline Semestre & $\begin{array}{c}\text { Equipos a líder de } \\
\text { turno }\end{array}$ & $\begin{array}{c}\text { Líder de turno a líder } \\
\text { de semestre }\end{array}$ & $\begin{array}{c}\text { Líder de semestre a } \\
\text { líder de carrera }\end{array}$ & $\begin{array}{c}\text { Líder de carrera a } \\
\text { Consejo administrativo }\end{array}$ \\
\hline Primero & 3 de cada mes & Del 3 al 7 & 8 & \\
Segundo & 9 decada mes & Del 9 al 13 & 14 & Entrega final, \\
Tercero & 15 de cada mes & Del 5 al 9 & 20 & Noviembre 13, 2020 \\
Cuarto & 21 de cada mes & Del 21 al 15 & 26 & \\
Quinto & 27 de cada mes & Del 27 al 1 & 2 & \\
\hline
\end{tabular}

Fuente: elaboración propia 
Drive. Por medio de carpetas compartidas se hará entrega de:

Avances.

Manual de Ejercicios Prácticos.

Video tutorial.

De acuerdo a fechas establecidas. Tabla 4.

\section{RESULTADOS}

A continuación, se presentan los resultados obtenidos tras la realización del estudio de caso: Material digital para la Tutoría entre pares, modalidad virtual.

\subsection{Proceso en base al Modelo de Chickering y Gamson (1987). Buenas Prácticas.}

Principio 1: Promover las relaciones entre los prestatarios de servicio social participantes.

Las sesiones virtuales con el esquema de Tutoría entre pares, permitió la formulación de objetivos claros, medibles y alcanzables, así como la elaboración de planes de trabajo comunes entre los equipos de producción y los Tutores líderes lo cual definió la responsabilidad de cada miembro en el trabajo colaborativo y se contó con la inmediatez de la retroalimentación en la interacción del grupo.

Principio 2: Desarrollar dinámicas de colaboración entre los participantes. Mediante la asignación de actividades, en base a objetivos definidos y planificados en el tiempo y la formación de equipos, a quienes se les programaron sesiones virtuales para generar espacios de interacción virtual, se favoreció el desarrollo de la dinámica de colaboración entre los participantes, que, de acuerdo a Barkley, et al (2007) promueve la reflexión, el intercambio y la revisión entre pares.

Principio 3: Aplicar técnicas activas para el aprendizaje. Se aplicó la metodología tutoría entre pares y trabajo colaborativo. En el aprendizaje colaborativo se enfatizó: el pensamiento crítico, la resolución de problemas y la construcción del conocimiento, coincidiendo con Prieto (2006) quien señala que el aprendizaje activo es un enfoque que busca superar la posición pasiva de los estudiantes y ubicarlos en situaciones que ponen en juego habilidades para la resolución de actividades con el propósito de lograr su implicación y protagonismo en ellas.

Principio 4: Permitir procesos de retroalimentación. Se contó con sesiones virtuales de tutoría entre pares para orientación y consulta, contactos de comunicación definidos y claros, así como retroalimentación en las evaluaciones de los avances tanto del manual como de los videos tutoriales de aprendizaje, brindando información a los prestatarios de servicio social durante todo el proceso y no solo al final del mismo.

Principio 5: Enfatizar el tiempo dedicado a la tarea. El establecimiento de objetivos y la planeación de actividades dieron a conocer a todos los participantes el trabajo a realizar, la calidad requerida en base a estándares, formatos y rúbricas y el tiempo disponible. Se establecieron sesiones virtuales semanales para generar un espacio de interacción del cual partir como tiempo dedicado a la tarea.

Principio 6: Comunicar altas expectativas. Desde la presentación del Proyecto se mostró una actitud motivadora y de confianza por parte del Consejo y de los tutores pares Líderes de carrera hacia todos los prestatarios de servicio social participantes y esta actitud prevaleció en toda la estructura organizacional y durante todo el tiempo que duró el proyecto lo cual generó un sentimiento de responsabilidad mutua y permitió alcanzar los objetivos establecidos.

Principio 7: Respetar la diversidad. La metodología tutoría entre pares por su naturaleza respeta la diversidad y las investigaciones señalan que proporcionan beneficios a todos los involucrados en aspectos de mejora cognitivas y socioemocionales en diversas áreas y entornos, así como muestran correlación positiva con resultados académicos, habilidades sociales, autoestima y hábitos de estudio. (Topping, 2005; Alzate y Peña, 2009; Arco y Fernández, 2011; Sánchez, 2014).

\subsection{Producción de Material digital: Carrera Contador Público.}

Con la asesoría de un equipo de 2 Tutores pares líderes de carrera, 10 Tutores pares líderes de semestre y 20 Tutores pares 
Tabla 5. Resultados de la carrera de Contador Público

\begin{tabular}{lccccc}
\cline { 2 - 6 } líderes de & Semestre & $\begin{array}{c}\text { Prestatarios } \\
\text { Servicio Social }\end{array}$ & Equipos & Materias & $\begin{array}{c}\text { Manuales y } \\
\text { videos }\end{array}$ \\
\cline { 2 - 6 } turno, & 1 & 32 & 15 & 2 & 15 \\
mediante & 2 & 31 & 15 & 3 & 15 \\
registros de & 3 & 32 & 15 & 2 & 15 \\
archivo, la & 32 & 15 & 2 & 15 \\
entrevista & 4 & $\mathbf{2 9}$ & 14 & 1 & 14 \\
directa y la & 5 & $\mathbf{1 5 6}$ & $\mathbf{7 4}$ & $\mathbf{1 0}$ & $\mathbf{7 4}$ \\
observación & \multicolumn{2}{c}{ Total } & & &
\end{tabular}

objetivo de

producción de 74 Manuales de ejercicios prácticos digitales y videos tutoriales de aprendizaje de materias prácticas de primero a quinto semestre en la carrera de Contador Público, como se muestra en la tabla 5.
Contador Público y 105 para la carrera de Licenciado en Administración, su alcance materias prácticas vigentes de primero a quinto semestre elaborados en el ciclo Junio Diciembre de 2020 en la FACPYA, UANL, de 105 Manuales de ejercicios prácticos digitales y videos tutoriales de aprendizaje de materias prácticas de primero a quinto semestre en la carrera de Contador Público, como se muestra en la tabla 6 .

Tabla 6. Resultados de la carrera de Licenciado en Administración

\begin{tabular}{ccccc}
\hline Semestre & $\begin{array}{c}\text { Prestatarios } \\
\text { Servicio Social }\end{array}$ & Equipos & Materias & $\begin{array}{c}\text { Manuales y } \\
\text { videos }\end{array}$ \\
\hline 1 & 35 & 18 & 2 & 18 \\
2 & 43 & 20 & 2 & 20 \\
3 & 43 & 19 & 2 & 19 \\
4 & 45 & 28 & 3 & 28 \\
5 & 49 & 20 & 1 & 20 \\
\hline Total & $\mathbf{2 1 5}$ & $\mathbf{1 0 5}$ & $\mathbf{1 0}$ & $\mathbf{1 0 5}$ \\
\hline
\end{tabular}

Fuente: elaboración propia

\subsection{Producción de Material digital:} Licenciado en Administración.

Con la asesoría de un equipo de 2 Tutores pares líderes de carrera, 10 Tutores pares líderes de semestre y 30 Tutores pares líderes de turno se alcanzó el objetivo de producción

Con una gran empresa de 445 prestatarios de servicio social y basados en las proposiciones teóricas de tutoría en pares, trabajo colaborativo y los principios de buenas prácticas, damos respuesta a nuestra pregunta de investigación ¿Cómo producir el material digital necesario para la tutoría entre pares, modalidad virtual? El logro, la producción de 74 Manuales de ejercicios prácticos digitales y videos tutoriales de aprendizaje para la carrera

\section{CONCLUSIÓN}

Este estudio de caso ofrece la oportunidad de observar el potencial conjunto e innovador de las proposiciones teóricas : Tutoría entre pares, trabajo colaborativo y principios de buenas prácticas de Chickering y Gamson (1987) en el proceso de producción de material digital para un Programa de Tutoría entre pares, modalidad virtual, con la participación de estudiantes prestatarios de servicio social, en modalidad virtual de la FACPYA, UANL, en un contexto complejo, debido a la pandemia COVID 19 y la migración obligada a la educación virtual, en el ciclo Junio - Diciembre de 2020. 
La tutoría entre pares permite $\mathrm{y}$ fomenta un mayor volumen de actividad en donde el estudiante tutor par líder y los estudiantes tutorados prestatarios de servicio social, como protagonistas guiados por profesionales, amplían sus respectivos conocimientos declarativos, sus capacidades de procedimiento y aplicación, mediante la construcción participativa y conjunta en sus interacciones virtuales, formando un círculo virtuoso que termina con el logro de los objetivos propuestos. Los resultados son consistentes con estudios previos sobre implementaciones de tutoría entre pares en otros contextos y áreas de estudio (Duran \& Flores, 2015; Duran, Flores, Mosca \& Santiviago, 2014; González-Fernández, García-Ruiz \& Ramírez-García, 2015).

El trabajo colaborativo implica la creación de grupos reducidos para trabajar, construir, aprender, cambiar y mejorar juntos, maximizando el aprendizaje de todos los involucrados. Coincidimos con Marcelo et. al., (2010) en que algunos de los aspectos clave para la efectividad de la colaboración son: Contar con metas estructuradas; Responsabilidad individual y compartida; conocimiento del progreso o avance; $y$ liderazgo compartido. Se confirma en base a nuestros resultados que el trabajo colaborativo es un método de interacción social efectivo, a pesar de que en este caso toda interacción se realizó de manera virtual, que se centra en los estudiantes trabajando juntos y compartiendo la responsabilidad en un proceso continuo de evaluación. (Cenich y Santos, 2006).

La incorporación de los principios de buenas prácticas de Chikering y Gamson (1987) en la planificación, diseño, e implementación del proceso de producción de material digital para el Programa de Tutoría entre pares, modalidad virtual, permite vigilar aspectos relevantes como: Promover las relaciones entre tutores pares líderes y tutorados; desarrollar dinámicas de colaboración entre los estudiantes; aplicar técnicas activas; permitir procesos de retroalimentación; enfatizar el tiempo de dedicación a la tarea; comunicar altas expectativas; y respetar la diversidad. Aspectos que pudieran ser obviados en la práctica sino es que se establecen como base del proceso. Los resultados son consistentes a estudios previos sobre educación presencial y virtual (Babb, Stewart y Johnson, 2013; Dixon, 2012; y Cakiroglu, 2014).

En cuanto al objetivo de la investigación: Describir un estudio de caso sobre el proceso de producción de material digital como estrategia emergente para migrar a la modalidad virtual de un Programa de Tutoría en pares, se confirma que utilizar las proposiciones teóricas: Tutoría entre pares, Trabajo colaborativo y los principios de buenas prácticas permite dirigir con efectividad una gran empresa formada por 2 administrativos y 445 estudiantes prestatarios de servicio social en la modalidad virtual, y alcanzar la producción de 179 Manuales de Ejercicios prácticos y Videos tutoriales de aprendizaje para las carreras de Contador Público y Licenciado en Administración, de primero a quinto semestre, en materias prácticas vigentes en la FACPYA, UANL durante el ciclo Junio - Diciembre 2020.

Este estudio de caso solo pretende presentar una experiencia que pueda inspirar a los profesores a la extrapolación a las aulas de las proposiciones teóricas: Tutoría entre padres, Trabajo colaborativo y Principios de buenas prácticas, de forma conjunta e innovadora para desarrollar actividades centradas en el estudiante que permitan, el aprendizaje activo y significativo, a fin de responder a los nuevos escenarios en la educación superior. Queda en el futuro una posible investigación, sobre la evolución del trabajo individual y colaborativo y el desarrollo de competencias, desde la perspectiva y opinión de los estudiantes participantes. 


\section{REFERENCIAS}

Alexander, R. (1997). Policy and practice in primary education: Local initiative, national agenda. London: Routledge.

Alzate, G., Peña, L. (2009). La tutoría entre iguales: una modalidad para el desarrollo de la escritura en la educación superior. Revista Universitas Psychologica, vol. 9, núm. 1. ISSN 657-9267.

Apaza, C., Seminario, R., Santa-Cruz J (2020) Factores psicosociales durante el confinamiento por el Covid-19 - Perú. Revista Venezolana de Gerencia. ISSN: 1315-9984. Vol. 25, núm. 90. Univesidad de Zulia Venezuela.

Arco, J.L., Fernández, F. (2011) Eficacia de un programa de tutorías entre iguales para la mejora de los hábitos de estudio del alumnado universitario. Revista de Psicodidáctica, vol. 16, núm. 1. ISSN: 1136-1034.

Asociación Nacional de Universidades e Instituciones de Educación Superior (ANUIES)-UNESCO. (2004). La educación superior virtual en América Latina y el Caribe. México: Serie Memorias. Colección Biblioteca de la Educación Superior. ANUIES, UNESCO, IESALC.

Babb, S., Stewart, C., y Johnson, R. (2013). Applying the seven principles for good practice in undergraduate education to blended learning environments. Practical applications and experiences in K-20 blended learning environments, pp.192.

Bangert, A.W. (2004) The seven principles of good practice: A framework for evaluating on-line teaching. The Internet and Higher Education, 7(3), 217-232.

Bonilla, L. A. G. (2016). Deliberación entorno a la Educación Virtual. Interconectando Saberes, (1), 77-89. http://is.uv.mx/index.php/IS/article/view/1112

Cakiroglu, U. (2014). Evaluating students' perspectives about virtual classrooms with regard to Seven principles of good practice. South African Journal of Education, 34(2), 1-19.

Cenich, G. y Santos, G. (2009) Aprendizaje significativo y colaborativo en un curso online de formación docente. Revista Electrónica de Investigación en Educación en ciencias. REIEC. Vol. 4 núm 2, pp 7-23.

Chickering, A. W. y Gamson, Z. F. (1987). Seven principles for good practice in under- graduate education. AAHE Bulletin, 39(1), 3-7.

Coffield, F. y Edward, S. H. (2009). Rolling out good, best and excellent practice What next? Perfect practice? British Educational Research Journal, 35(3), 371-390.

Coll, C., (2008) Psicología de la educación virtual: aprender y enseñar ccon las tecnologías de la información y la comunicación. Madrid: Ediciones Morata.

Collazos, C. y Mendoza, J. (2006) Cómo aprovechar el aprendizaje colaborativo en el aula. Educación y Educadores. Vol. 9 , núm. 2, pp 61-76. ISSN: 0123-1294.

Clark, I. y Dumas, G. (2015) Toward a neural basis for peer-interaction: what makes peer-learning tick? Frotiers in Psychology, 6, 1 -12 doi 10.3389/fpsyg.2015.00028.

De Pablos, J. y González, T. (2007). Políticas educativas e innovación educativa apoyada en TIC: sus desarrollos en el ámbito autonómico. Actas de las II Jornadas Internacionales sobre política educativa para la sociedad del conocimiento. Granada.

De Pablos, J. y Jiménez, R. (2007). Buenas prácticas con TIC apoyadas en las políticas educativas: Claves conceptuales y derivaciones para la formación en competen- cias ECTS. Revista Latinoamericana de Tecnología Educativa, 6(2), 15-28. 
Dixon, M. D. (2012). Creating effective student engagement in online courses: What do students find engaging? Journal of the Scholarship of Teaching and Learning, 10(2), 1-13.

Duran, R., Estay-Niculcar Ch.(2016) Formación en buenas prácticas docentes para la educación virtual. RIED. Revista Iberomericana de Educación a Distancia, 19 (1) 209 -232.

Duran, D., Flores, M. ( 2015 ). Prácticas de tutoría entre iguales en universidades del Estado Español y de Iberoamérica. REICE. Revista Iberoamericana sobre calidad, eficacia y cambio en educación, vol. 13, núm. 1. ISSN 1696-4713.

Duran, D., Flores, M., Mosca, A. y Sabtuvuagim C. (2014). Tutoría entre iguales del concepto a la práctica en las diferentes etapas educativas. InterCambios, 2(1), 31-39. Disponible en: http://grupsderecerca.uab. cat/grai/sites/grupsderecerca.uab.cat.grai/files/art3_duran.pdf

Epper, R. y Bates, A. (2004). Enseñar al profesorado cómo utilizar la tecnología. Buenas prácticas de instituciones líderes. Barcelona: Editorial UOC.

Fernández, K. y Vallejo, A. (2014). La educación en línea: Una perspectiva basada en la experiencia de los países. Revista de Educación y Desarrollo, 29, 29-39.

García, L. (1999). Historia de la educación a distancia. Revista Iberoamericana de Educación a Distancia (RIED), 2(1), 11-40.

Gavilán, P. (2009) Aprendizaje cooperativo. Papel del conflicto sociocognitivo en el desarrollo intelectual. Consecuencias Pedagogicas. Revista Española de Pedagogía. LXVII, núm. 242, pp 131-148.

González, N., García, R. y Ramírez, A. (2015) Aprendizaje cooperativo y tutoría entre iguales en enornos virtuales universitarios. Estudios Pedagogicos . Vol. 41(1), pp 111-124. ISSN: 0716050X.

Graham, C., Cagiltay, K., Lim, B., Craner, J. y Duffy, T. M. (2001). Seven principles of effective teaching: A practical lens for evaluating online courses. The Technology Source, 30(5), 50.

Gros, B. (2012) Retos y tendencias sobre el futuro de la investigación acerca del aprendizaje con tecnologías digitales. RED. Revista de Educación a Distancia. 3 (2) , 1 - 13.

Hutchins, H. M. (2003). Instructional immediacy and the seven principles: Strategies for facilitating online courses. Online Journal of Distance Learning Administration, 6(3), 1-13.

Leyes y Reglamentos de la Universidad Autónoma de Nuevo León. Reglamento de Servicio Social. (2014). http: uanl.mx/en linea/institucional.

Levin, R. y Rubin, D. (1996) Estadística para Administradores . 6ª Edición. México. Editorial Prentice. Hall Hispanoamericana.

Luján-Piedrahita, M. (2020) Virtualidad en el curso teórico de Medicina Interna en estudiantes de V, VI y VII semestre a raíz de la pandemia COVID-19 durante el primer semestre del 2020. Medicina U.P.B., ISSN: 2357-6308 vol. 39 , núm. 2, pp. 66-72

Luzón, A., Porto,M., Torres, M., Ritacco, M.(2009)Buenas prácticas en los programas extraordinarios de atención a la diversidad en centros de educación secundaria. Una mirada desde la expetiencia. Revista de Curriculum y Formación del Profesorado, 13 (3), 217-238.

Marcelo, C., Mayor, C. y Gallego, B. (2010) Innovación educativa en España desde el punto de vista de sus protagonistas. Revista de Curriculum y Formación del Profesorado 14 (1), 111 - 134.

Marciniak, R., y Gairín-Sallán, J. (2018). Dimensiones de evaluación de calidad de educación virtual: revisión de modelos referentes. RIED. Revista Iberoamericana de Educación a Distancia, 21(1), 217-238. https://doi.org/10.5944/ried.21.1.16182 
Menéndez, J.L. (2011) Fundamentos de un Programa de Tutorías entre Iguales en los estudios universitarios de las artes. OBSERVAR. 5, 42-67. ISSN: 1988-5105.

Mkpanang, J.L. (2016) Efectos de las estrategias de tutorías en la clase y reciprocas sobre el rendimiento de problemas matemáticos de los estudiantes en conceptos de electricidad en la física. Revista Internacional de Educación, Aprendizaje y Desarrollo. Vol. 4 (3), 37-44.

Pérez Serrano, G. (1994) Investigación cualitativa. Retos, interrogantes y métodos. España. La Muralla.

Piaget, J. ( 1983 ) La Psicología de la Inteligencia. Barcelona. Crítica.

Pineda, E., Alvarado, E., Canales, F. (1994) Metodología de la Investigación: Manual para el desarrollo del personal de salud. $2^{\mathrm{a}}$. Edición. Washington DC:OPS.

Rama, C. A. (2014). University virtualisation in Latin America. RUSC. Universities and Knowledge Society Journal, 11(3), 32-41.

Ruiz, G. (2020) Covid.19. Pensar la educación en un escenario inédito. RMIE. Revista Mexicana de Investigación Educativa. ISSN: 2594-2271. Vol. 25, núm. 85 pp 229-237.

Sánchez, M. (2014) La tutoría entre iguales como estrategia educativa para desarrollar competencias. Revista electrónica de Psicología Iztacala, vol. 17, núm 1. Universidad Autónoma de México.

Silvio, J. (2006). Hacia una educación virtual de calidad, pero con equidad

y pertinencia. Revista de Universidad y Sociedad del Conocimiento, 3(1),

1-14. añosdespuésdelaConferencia0Mundialde1998. UNESCO.IESALC.

Stake, R.E. (2005) Investigación con estudio de casos. Madrid, Morata.

Suárez, J. M. y Anaya, D. (2004). Educación a distancia y presencial: Dife- rencias en los componentes cognitivo y motivacional de estudiantes universitarios. RIED. Revista Iberoamericana de Educación a Distancia, 7(1-2), 65-75.

Tobin, T. J. (2004). Best practices for administrative evaluation of online faculty. Online Journal of Distance Learning Administration, 7(2). Disponible en http://www.westga.edu/ distance/ojdla/summer72/tobin72.html.

Topping, K.J. (2015) Peer tutoring: old method, new developments. Journal for the study of Education and Development. 38(1) 1-29.

Tooping, K.J. (2005) Trends in peer learning. Educational Psychology, 25(6), 631-645.

Torrado, D., Manrique, E., Ayala, J. (2016) La tutoría entre pares: una estrategia de enseñanza y aprendizaje de histología en la Universidad Industrial de Santander. Medicas UIS, vol. 29, núm. 1. Bucaramanga. ISSN 0121-0319.

Tunnermann, C. (2008) La educación superior en América Latina y el Caribe. Diez años después de la Conferencia Mundial de 1988. UNESCO.IESALC.

Viáfara, J., Ariza, J. (2008) Un modelo tutorial entre compañeros como apoyo al aprendizaje autónomo del inglés. Ikala. Revista de lenguaje y cultura, vol. 13, núm. 19. Universidad de Antioquia, Medellín, Colombia. ISSN 012.

Vygotsky, L.S. ( 1984 ) Aprendizaje y desarrollo intelectual en la edad escolar. Infancia y Aprendizaje. 27-28: 3-4, pp105 - 116.

Zabalza, M. (2012). El estudio de las buenas prácticas docentes en la enseñanza universitaria. Revista de Docencia Universitaria, 10(1), 17-42 